\title{
An Algorithm for Computing Solutions to the Range Limited Routing Problem Using Electrical Trucks
}

\author{
YAVUZ GUNALAY \\ Faculty of Economic and Administrative Sciences \\ Bahcesehir University \\ 34353 Besiktas Istanbul, Turkey \\ TURKEY \\ yavuz.gunalay@bahcesehir.edu.tr \\ JULIAN SCOTT YEOMANS \\ OMIS Area, Schulich School of Business \\ York University \\ 4700 Keele Street, Toronto, ON, M3J 1P3 \\ CANADA \\ syeomans@,schulich.yorku.ca
}

\begin{abstract}
Increasing urban intensification has caused civic planners to devote additional resources to more appropriate logistics planning. Electric vehicles have proved to be both a lower cost alternative and more environmentally friendly than the more ubiquitous internal combustion engine vehicles. However, prevailing decision-making formulations employed by municipalities and businesses are not necessarily computationally conducive for the evaluation and optimization of urban transportation systems using electric vehicles. An innovative computational approach, the range limited routing problem, is introduced that enables urban planners to more readily evaluate the contributions of electric vehicles to the city logistics decision-making process. While there is no generalized solution technique for solving this new formulation, this paper employs the Firefly Algorithm (FA) metaheuristic to solve the range limited routing problem using electric trucks.
\end{abstract}

Keywords: - Urban Logistics, Electric Vehicles, Transshipment Problem, Firefly Algorithm

Received: September 13, 2019. Revised: February 24, 2020. Accepted: March 11, 2020. Published: March 27, 2020.

\section{Introduction to Urban Logistics and Electric Vehicles}

Finding a solution to urban freight transshipment problems is an important aspect of sustainable development planning within large muncipalities [1][2]. Effective civic transportation infrastructure must combine account for many incongruent constraints arising from vehicle congestion, high fuel usage, lack of human resources, stuctural deficiencies, and various prevailing environmental components [3]-[5]. A major sub-field in logistics planning has emerged to address recently introduced environmental requirements [6][7]. To satisfy these sustainability constraints, urban freight carriers must simultaneously integrate low-cost, just-in-time transportation systems into high levels of customer satisfaction [8].

All logistics activities occur under the numerous constraints and limitations inherent to cities. In addition to market-economic benefits, urban planning must simultaneously achieve social, sustainable, and environmental satisfaction [5][9].
The goal uniting freight transport with urban logistics is to optimize logistics operations within the cities under social, environmental, energy usage, economic, traffic congestion, and financial constraints $[2][3][5][8]$. Without an effective logistics plan, civic development will not improve the overall quality of life. Therefore, effective urban logistics is one of the key building-blocks for successful urban planning [6][10].

Street-level noise pollution in combination with vehicle emissions represent two of the foremost environmental problem areas in urban logistics planning [3][5]. Although there has been a significant decrease in vehicle emissions due to more stringent government regulations, total emissions have actually increased due to even higher traffic volumes [1][11]. While rural air quality has improved, air quality has remained a major obstacle on urban roadways [12][13]. Consequently, further actions must be taken to improve vehicle emission impacts on human health [5]. Noise pollution is also a major cost arising from 
transportation [14]. Heavy trucks, buses, dieselengine cars, and motorcycles all contribute to elevated urban noise pollution. Replacing internal combustion engines (ICE) with silent-running, zeroemission electric motors affords one primary mechanism for overcoming excessive emissions issues and urban noise levels [12][13][15][16]. Recent developments in electric storage and automotive technology have resurrected awareness for employing electric vehicles (EV) to conduct urban freight delivery [15][17]. Relative to ICE vehicles, EVs possessing high efficiency electric motors hold numerous advantages such as low fuel and maintenance costs, zero emissions (improves air quality while decreasing emissions), and silent operations issues [9][15][16].

Unfortunately, if a decision between selecting ICE and EV engine vehicles for urban transshipment is posed as a strict either-or evaluation, using existing computational methods, it is not difficult to establish that ICE vehicles would be the preferred choice. In such comparative decision-making assessments, new EV technology currently lacks widespread commercial acceptance due to low vehicle range, long recharge times, and significant initial adoption costs [15][17]. Thus, the introduction of significant technological advances are requisite prior to considering EVs as an effective replacement in long-range transportation [2]. Nevertheless, while it may not currently be feasible for EVs to act as a comprehensive substitute for the logistical satisfaction of long-haul heavy-truck transportation, it will be computationally demonstrated that room does exist for the partial deployment of EVs in urban logistics. Low operation costs do render EVs as an attractive alternative to ICE vehicles for within-city transportation [1][12]. Specifically, although EVs may not appear feasible for universal transportation purposes, when some routes can be electrified, it can be shown that a significant cost reduction can exist. Thus, municipalities and companies should ascertain which subsets of civic routes are amenable to electrification. Under appropriate conditions, the urban transshipment problem with smart vehicles can be modified into a detemination of what proportion of the transportation networks ought to be served by EVs and ICE vehicles, respectively, and an identification of exactly which urban routings each vehicle type should cover. In this study, new mathematical model of this adapted urban transshipment problem with $\mathrm{EVs}$ is formulated. Because the actual optimization of this formulation can prove challenging, the computationally efficient Firefly Algorithm (FA) metaheuristic is employed for its solution [18][19]. In this paper, the computational effectiveness of this innovative optimization approach for solving the urban transshipment problem is established on an example of the range limited routing problem.

\section{Electric Vehicle Selection Issues}

Depending upon the specific configurations, the overall vehicle expenses of most EVs will tend to be $20 \%$ to $50 \%$ higher than ICE vehicles. The increased costs arise mostly from the Lithium-ion batteries, although the costs of other components, especially the engines, are currently much higher than ICE motors. Conversely, as there is only a one rotating part in an electric motor in comparison to numerous moving parts in a combustion engine, the EV maintenance costs are approximately half those of ICE engines [15][17]. Moreover, EV fuel costs are considerably lower than ICE vehicles. Depending upon the fuel and electricity prices, cost per kilometre for EVs is from one half (USA) to one-sixth (Turkey). The environmental impact of EVs depends heavily on the generation source of electricity (e.g. wind, solar, hydroelectric, coal, nuclear). However, there is still an improvement in carbon emissions even when the electricity is sourced from coal. EVs area always far superior to ICE trucks with respect to air quality when measured at street level, [12].

The technology of ICE vehicles is far more versatile for long-range transportation purposes than that supplied by existing batteries. For example, petroleum-based vehicles can be refueled swiftly in less than 5 minutes and then be driven for distances of exceeding $500 \mathrm{~km}$. On the other hand, the batteries of a standard EV take more than an hour to recharge, providing only sufficient sufficient capacity to cover a range of no more than 100-150 $\mathrm{km}$. While swapping a battery or replenishing its electrolyte fluid provide two alternative approaches to the recharging speed dilemma, the battery distance capacity limitations do not change. Thus, for long range transportation needs, recharging points for EVs would need to be planned along the route to perhaps coincide with scheduled driver rest periods. The European Commission [4] restricts the longest nonstop period of truck driving to 4.5 hours which must then be followed by a requisite break of 45 minutes. Accordingly, to satisfy the EU driving requirements, long-range EV freight transportation would need to have a range of approximately 400 $\mathrm{km}$ together with a battery that could be fully charged within an hour. In such a situation, all travel and rest periods would have to be highly 
synchronized with battery recharging times, thereby introducing significant operational challenges and additional logistical planning in order to co-ordinate long-range transportation activities.

In major urban environments, EV distance limitations are a less challenging problem than the actual battery costs. For municipal logistics, EV recharging periods could occur during regular onsite loading/unloading activities or during preprogrammed periods of downtime (i.e. overnight). Regrettably for $\mathrm{EV}$, in large cities where $100^{+} \mathrm{km}$ routes would not be uncommon, the $100-150 \mathrm{~km}$ distance constraint would impose significant operational boundaries on the planning requirements. Additionally, to deliver refridgerated products, the overall distance limitations of EVs would be even more restricted due to the extra loading drawn from the batteries.

Therefore, if the vehicle selection problem is posed as an either-or determination between ICE technology and EVs, current decision-making models would select ICE transportation in all urban transshipment situations. However, a different solution approach arises if the problem is posed as determining the optimal proportion of EVs to include in the transportation fleet.

\section{Example Problem with Electric Vehicles}

To facilitate a better understanding of the ensuing modelling, this section introduces an easyto-solve urban transshipment problem with electric vehicles. Consider a symmetrical, urban distribution network containing a centralized storage warehouse, $A$, that supplies the four outlying facilities $B, C, D$, and $E$ (Figure 1). For simplicity, assume that the demand at each store is 1 unit/day and that this quantity is delivered daily by a truck with a capacity of 2 units. Assume that the transportation and environmental costs are proportional to the distance covered and independent of the load amount. Hence, while in this problem, only operational costs are provided, in "real life", the calculations would also need to incorporate the full spectrum of expenses including environmental charges. From Figure 1, to satisfy the requisite transshipment requirements, it can be ascertained that the two feasible routes $A-E$ $A-C-A$ and $A-B-A-D-A$ would have a combined cost $160+160=320$ units, while routes $A-D-C-A$ and $A$ $E-A-B-A$ would cost $180+120=300$ units. Nevertheless, it is relatively straightforward to determine that the minimum cost routes, $A-E-D-A$ and $A-C-B-A$, possess a total cost of $120+120=$ 240 units.

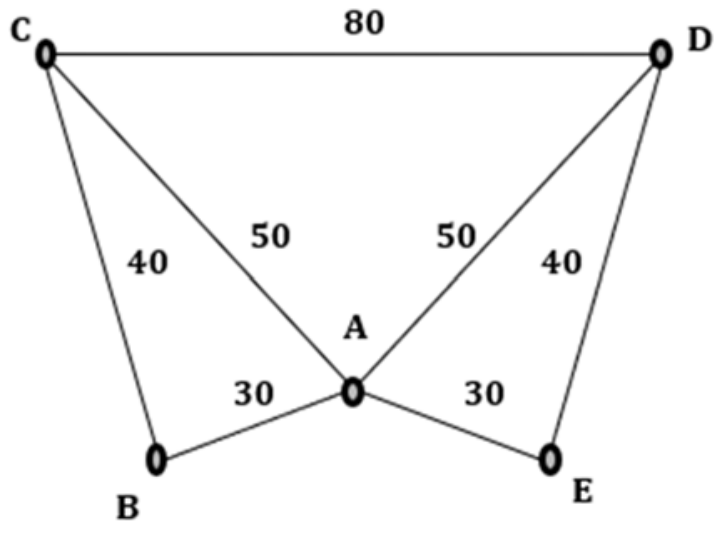

Figure 1. Sample Distribution Network (The numbers denote the distance or cost between nodes)

If the entire truck fleet could be replaced by EVs with $1 / 6$ fuel costs, the minimum total cost would decrease to only $240 / 6=40$ units. However if a reasonable cost electric truck typically possesses a $100 \mathrm{~km}$ range, then it would be neither operationally feasible to undertake $120 \mathrm{~km}(A-C-B-A, A-E-D-A)$ delivery routes, nor possible to even service the facilities $C$ and $D$. Due to these infeasibilities, if the problem were posed as whether the ICE trucks should be resplaced by more energy efficient, environmentally benign EVs, the response would necessarily be negative.

However, if the problem was rephrased as finding "the ratio of the fleet to be converted to EVs" then this would lead to a completely different decision-problem. Specifically, the question could be re-stated as "in order to reduce overall costs, which routes could be satisfied by EVs and which routes would be served by ICE trucks?". In the transshipment network if nodes $B$ and $E$ were served by EVs, while $C$ and $D$ were still serviced by ICE trucks, then the optimal routes $A-E-A-B-A$ and $A-D$ $C$ - $A$ would now have a total cost of 200 units $(180+$ 120/6). Thus, it can be seen that a partial coverage of the distribution network using EVs would have better overall costs than the originally determined 240 units. In this situation, the urban transshipment problem with electric vehicles converts into a determination of what proportions of the network are served by EVs and ICE vehicles, and exactly which routes do these respective vehicle types need to cover. Optimizing this version of the urban transshipment problem with EVs will, henceforth, be referred to as the Range Limited Routing Problem (RLRP). 


\section{Range Limited Routing Problem Formulation}

A generalized mathematical formulation of the RLRP will be provided in this section. Together with the additional challenges that arise from the range limitations of EVs, the approach for formulating the RLRP requires a combination of both standard vehicle routing problems [20] and capacitated vehicle routing problems [21].

The following notation will be employed in the RLRP:

$k$-index for trucks $k=1, \ldots, K$.

$i, j-$ index for node (stores: $1, . ., N$ and warehouse: 0 ).

$R_{k}$ and $T_{k}$ - represents truck range and capacity, respectively, for truck $k, k=1, ., K$.

$d_{0}$ - warehouse capacity.

$d_{i}-$ demand at node $i, i=1 . ., N$.

$r_{i j}$ - distance between node $i$ and $j, i, j=0, . ., N$.

$l_{i k}-$ load transshipped to node $i$ using truck $k$.

One assumption for the RLRP is that total capacity must equal the total demand with no excess capacity:

$$
\sum_{k=1 t o K} T_{k}=\sum_{i=1 t o N} d_{i}
$$

Hence, the complete mathematical programming formulation of the RLRP is to determine a solution to the following problem:

$$
\operatorname{Max} \sum_{k=1 t o K} \sum_{i=0 t o N} \sum_{j=i+1 t o N} d_{j} x_{i j k}
$$

Subject to:

$$
\begin{array}{ll}
\sum_{k=1 t o K} \sum_{j=1 t o N} x_{0 j k} \leq K & \\
\sum_{i=0 t o N, i \neq j} x_{i j k}=\sum_{j=0 t o N, i \neq j} x_{i j k} & \forall j, k \\
\sum_{j=1 t o N, i \neq j} x_{i j k} \leq 1 & \forall i, k \\
\sum_{i=1 t o N, i \neq j} x_{i j k} \leq 1 & \forall j, k \\
\sum_{i=0 t o N} \sum_{j=0 t o N, i \neq j} r_{i j} x_{i j k} \leq R_{k} & \forall k \\
l_{j k} \leq d_{j} \sum_{i=1 t o N, i \neq j} x_{i j k} & \forall j, k \\
\sum_{k=1 t o K} l_{i k} \leq d_{i} & \forall i \\
\sum_{i=1 t o N} l_{i k} \leq T_{k} & \forall k
\end{array}
$$

The constraints in the model ensure that at most $K$ trucks are used to deliver the goods (3), the truck routes are continuous and non-recurrent (4)-(6), the trucks do not exceed their range capacities (7), and that the demand at each store is satisfied by at least one truck (8)-(10). Determining a solution that satisfies all of the constraints enables a search for the feasibility of the urban transshipment problem with EVs. The optimal RLRP requires a feasible solution that maximizes the satisfied demand at each node and provides an urban cargo, fleet forming decision to the urban transshipment problem. Optimizing the RLRP can prove very computationally challenging. In the subsequent sections, the population-based, metaheuristic FA will be used to efficiently optimize the RLRP [22].

\section{Firefly Algorithm Optimization}

This section provides a brief overview of the mechancis of the FA procedure that is covered in significantly greater detail in [18][19][23]. The FA is a population-based metaheuristic. Each firefly in the population corresponds to one potential solution to a problem and the initial population of fireflies is distributed randomly and uniformly through the decision space. The FA operates under the following three concepts: (i) All fireflies within the population are considered unisex, implying that any one firefly could be attracted to any other firefly irrespective of their sex; (ii) The relative attraction between two fireflies is directly proportional to their respective brightness. This implies that for any two fireflies, the less bright firefly will be predisposed to advance towards the brighter one. However, brightness and attraction both decrease as the distance between the fireflies increases. A firefly will move about randomly, if no brighter firefly exists within its neighborhood of visibility; and, (iii) The relative brightness of a firefly is determined by the value of its objective function. For example, in a maximization problem, the brightness is proportional to the value of the objective function.

In the FA, there are two important aspects: the formulation of attractiveness and the variation of light intensity. For simplicity, firefly attractiveness is determined by its brightness which, in turn, is related to the value of its objective function. In the simplest case, the brightness of a firefly at a location $\boldsymbol{X}$ would be its calculated objective value $F(\boldsymbol{X})$. However, the attractiveness, $\beta$, between fireflies is relative and will vary with the distance $r_{i j}$ between firefly $i$ and firefly $j$. Light intensity decreases with the distance from the source, and light is also 
absorbed in the media, so the attractiveness needs to vary with the degree of absorption. Consequently, the overall attractiveness of a firefly is calculated by

$$
\beta=\beta_{0} \exp \left(-\gamma^{2}\right)
$$

where $\beta_{0}$ is the attractiveness at distance $r=0$ and $\gamma$ is the fixed light absorption coefficient for a specific medium. If the distance $r_{i j}$ between any two fireflies $i$ and $j$ located at $\boldsymbol{X}_{i}$ and $\boldsymbol{X}_{j}$, respectively, is calculated using the Euclidean norm, then the movement of a firefly $i$ that is attracted to another more attractive (i.e. brighter) firefly $j$ is determined by

$$
\boldsymbol{X}_{i}=\boldsymbol{X}_{i}+\beta_{0} \exp \left(-\chi\left(\mathrm{r}_{\mathrm{ij}}\right)^{2}\right)\left(\boldsymbol{X}_{i}-\boldsymbol{X}_{j}\right)+\alpha \boldsymbol{\varepsilon}_{i} .
$$

In this expression of movement, the second term is due to the relative attraction and the third term is a randomization component. Yang [18][19] indicates that $\alpha$ is a randomization parameter normally selected within the range $[0,1]$ and $\varepsilon_{i}$ is a vector of random numbers drawn from either a Gaussian or uniform (generally $[-0.5,0.5]$ ) distribution. It should be explicitly noted that this expression represents a random walk biased toward brighter fireflies and if $\beta_{0}=0$, it becomes a simple random walk. The parameter $\gamma$ characterizes the variation of the attractiveness and its value determines the speed of the algorithm's convergence. For most applications, $\gamma$ is typically set between 0.1 to 10 [19]. In any given optimization problem, for a very large number of fireflies $n>k$, where $k$ is the number of local optima, the initial locations of the $n$ fireflies should be distributed essentially uniformly throughout the search space. As the FA proceeds, the fireflies tend to converge into these local optima (including the global ones). By comparing the best solutions among all of these optima, the global optima can easily be determined. Yang [19] proves that the FA will approach the global optima when $n \rightarrow \infty$ and the number of iterations $t$, is set so that $t>>1$. In reality, the FA has been found to converge extremely quickly with $n$ set in the range 20 to 50 [18][19]. Furthermore, the FA can find both global optima and local optima concurrently, which contributes huge computational and efficiency advantages.

\section{Computing Solutions to the Range Limited Routing Problem with the Firefly Algorithm}

In this section, a computational testing of the efficacy of solving the RLRP using the FA metaheuristic will be illustrated. Since the example problem is small, assume that the direction of travel specified in the distribution network is considered non-commutative (i.e. the route $A-B-C-A$ is considered to be different from the route $A-C-B-A$ ). Under these problem specifications, the RLRP of the example problem was solved by the FA-driven metaheuristic to solution shown in Table 1. The computational example illustrates how the very computationally efficient, FA-based metaheuristic can be used to compute a solution to the RLRP. It can be explicitly noted that the best solution calculated by the FA procedure is identical to the optimal solution previously found in the RLRP example section.

\begin{tabular}{|l|l|l|}
\hline & $\mathbf{F}(\boldsymbol{X})$ & $\boldsymbol{X}$ (Truck Routes) \\
\hline Best Found & 200 & $(A-E-A-B-A),(A-D-C-A)$ \\
\hline
\end{tabular}

Table 1. Objective Value and Solution for the RLRP

As described earlier, the selection of EVs for support in urban transshipment planning cannot necessarily be stated in either-or terms with respect to the replacement of ICE trucks with EVs. Consequently, in addition to calculating the optimal proportion of EVs relative to ICE vehicles needed to service an urban logistics network, the RLRP model was formulated to also determine the optimal, specific truck routes for each type of vehicle. The computational results from the RLRP urban transshipment problem demonstrate that the FAdriven algorithm provides a highly suitable approach for producing the overall single optimal solution of the problem formulation.

\section{Conclusion}

While electric vehicles may not provide a direct substitute for the logistical requirements of longhaul heavy-truck transportation, space does exist for their employment in urban logistics. The decision to use electric vehicles can be made either through a strictly either-or evaluation or, instead, the ratio of partial electrification required in covering a subset of the urban routes. Although a complete conversion to EVs may not appear feasible, in general, when a portion of the routes can be electrified, there is a significant potential for cost reduction. Hence, to lower their costs, municipalities and companies need to determine which routes are suitable for electrification. The RLRP model formulates the problem, but determining its solution can prove computationally challenging. Therefore, an efficient 
solution approach to optimize the RLRP model is required.

This paper has employed the computationally efficient FA-based metaheuristic procedure to conduct this solution determination. The computational efficacy of employing the algorithm in conjunction with the population-based FA metaheuristic was demonstrated on the urban transhipment RLRP example. The computational procedure found the optimal solution to the formulated RLRP problem. The practicality of this FA-based computational approach for the urban transhipment problem using EVs can clearly be extended to wide range of other "real world" engineering and scientific applications. Such extensions will be examined in future research.

\section{References:}

[1] C. Thiel, W. Nijs, S. Simoes, J. Schmidt, A. van Zyl, and E. Schmid, The Impact of the EU Car CO2 Regulation on the Energy System and the Role of Electro-Mobility to Achieve Transport Decarbonisation, Energy Policy, Vol. 96, 2016, pp. 153-166.

[2] S. Winkelhaus, and E.H. Grosse, Logistics 4.0: A Systematic Review Towards a New Logistics System, International Journal of Production Research, Vol. 58, No. 1, 2020, pp. 18-43.

[3] A. De Marco, G. Mangano, and G. Zenezini, Classification and Benchmark of City Logistics Measures: An Empirical Analysis, International Journal of Logistics Research and Applications, Vol. 21, No. 1, 2018, pp. 119.

[4] European Commission. Panorama of Transport, 2009. Retrieved from https://ec.europa.eu/eurostat/documents/321749 4/5711595/KS-DA-09-001-EN.PDF/9c90d4895009-4acc-9810-ae39612897d3.

[5] M. Kiba-Janiak, and J. Witkowski, Sustainable Urban Mobility Plans: How Do They Work?, Sustainability, Vol. 11, No. 17, 2019, pp. 4605.

[6] E. Taniguchi, Concepts of City Logistics for Sustainable and Liveable Cities, Procedia Social and Behavioral Sciences, Vol. 151, No. 10, 2014, pp. 310-317.

[7] UNESCAP. Commercial Development of Regional Ports as Logistics Centres. United Nations. Economic and Social Commission for Asia and the Pacific (UNESCAP), 2003. Retrieved from https://www.unescap.org/sites/default/files/pub _2194_fulltext.pdf.
[8] E. Taniguchi, R.G. Thompson, T. Yamada, and R. van Duin, City Logistics, Holland: Pergamon, 2001.

[9] D.B. Richardson, Electric Vehicles and the Electric Grid: A Review of Modeling Approaches, Impacts, and Renewable Energy Integration, Renewable and Sustainable Energy Reviews, Vol. 19, No. 3, 2013, pp. 247-254.

[10] E. Taniguchi, and R.E.C.M. van der Heijden, An Evaluation Methodology for City Logistics, Transport Reviews, Vol. 20, No. 1, 2000, pp. 65-90.

[11] EEA Report, No. 3/2004, Ten Key Transport and Environment Issues for Policy-Makers, Copenhagen: European Environment Agency, 2004.

[12] M. Hernandez, M. Messagie, O. Hegazy, L. Marengo, O. Winter, and J. Van Mierlo, Environmental Impact of Traction Electric Motors for Electric Vehicles Applications, The International Journal of Life Cycle Assessment, Vol. 22, No. 1, 2017, pp. 54-65.

[13] R.N. Tuncay, and Ö. Üstün, Otomativ Sektör Kurulu Raporu: Elektrikli Araçlarda Geçmişten Geleceğe Bakış, MÜSİAD, 2012.

[14] European Commission. Road Transport: Reducing CO2 Emissions from Vehicles, 2016. Retrieved from http://ec.europa.eu/clima/policies/transport/vehi cles/index_en.htm.

[15] S. Khemakhem, M. Rekik, and L. A. Krichen, Flexible Control Strategy of Plug-In Electric Vehicles Operating in Seven Modes for Smoothing Load Power Curves in Smart Grid, Energy, Vol. 118, 2017, pp. 197-208.

[16] M. Weiss, M.K. Patel, M. Junginger, A. Perujo, P. Bonnel, and G. Grootveld, On the Electrification of Road Transport - Learning Rates and Hybrid-Electric and Battery Electric Vehicles, Energy Policy, Vol. 48, No., 20129, pp. 374-393.

[17] A.S. Brower, T. Kuramochi, M. Broek, and A. Faaij, Fulfilling the Electricity Demand of Electric Vehicles in the Long Term Future: An Evaluation of Centralized and Decentralized Power Supply Systems, Applied Energy, Vol. 107, No. 1, 2013, pp. 33-51.

[18] X.S. Yang, Firefly Algorithms for Multimodal Optimization, Lecture Notes in Computer Science, 5792, 2009, pp. 169-178.

[19] X.S. Yang, Nature-Inspired Metaheuristic Algorithms 2nd Ed., Frome (UK): Luniver Press, 2010.

[20] S.N. Kumar, and R. A Panneerselvam, Survey on the Vehicle Routing Problem and Its 
Variants. Intelligent Information Management, Vol. 4, No. 3, 2012, pp. 66-74.

[21] P. Toth, and D. Vigo, The Vehicle Routing Problem, USA: Society for Industrial and Applied Mathematics, 2002.

[22] G. Gunalay, and J. Yeomans, An Innovative Modelling and Decision-Support Approach for Evaluating Urban Transshipment Problems Using Electrical Trucks, Working Paper, 2020.

[23] G. Gunalay, and J. Yeomans, Multicriteria Generation of Alternatives for Engineering Optimization Problems Using PopulationBased Metaheuristics: A Computational Test, WSEAS Transactions on Computers, Vol. 18, No. 31, 2019, pp. 239-247. 